\title{
VLBI maser kinematics in high-mass SFRs: G23.01-0.41
}

\author{
Alberto Sanna ${ }^{1}$, Luca Moscadelli ${ }^{2}$, Riccardo Cesaroni ${ }^{2}$ \\ and Ciriaco Goddi ${ }^{3}$ \\ ${ }^{1}$ Max-Planck-Institut für Radioastronomie, Auf dem Hügel 69, 53121 Bonn, Germany \\ email: asanna@mpifr-bonn.mpg.de \\ ${ }^{2}$ INAF, Osservatorio Astrofisico di Arcetri, Largo E. Fermi 5, 50125 Firenze, Italy \\ email: mosca@arcetri.astro.it; cesa@arcetri.astro.it \\ ${ }^{3}$ European Southern Observatory, Karl-Schwarzschild-Strasse 2, D-85748 Garching bei \\ München, Germany \\ email: cgoddi@eso.org
}

\begin{abstract}
Very Long Baseline Interferometry studies of different maser species observed at multiple epochs allow complementary measurements of the 3-dimensional velocity field of gas close $\left(\lesssim 10^{3} \mathrm{AU}\right)$ to massive young stellar objects. Here, we review our recent results toward the high-mass star-forming region G23.01-0.41, where all the strongest molecular maser transitions known to date cluster within $2000 \mathrm{AU}$ from the center of an hot molecular core and are associated with a so called extended green object. The overall maser kinematics reveals a common outflowing motion from a central object; the details of the spatial distribution and velocity field of each maser species hint at the presence of different dynamical structures: a collimated jet, a wide-angle wind, and a flattened rotating core. We further compare the simultaneous presence of maser emission from different molecular species with a recent evolutionary sequence for masers associated with massive young stellar objects.
\end{abstract}

Keywords. masers, techniques: high angular resolution, stars: formation, stars: individual (G23.01-0.41).

\section{Introduction}

How do high-mass stars $\left(\mathrm{M}_{\text {star }}>8 \mathrm{M}_{\odot}\right)$ form? And what are the physical properties and dynamics of their environments locally? Maser emission in high-mass star-forming regions (HMSFRs) is a signpost of the earliest evolutionary phases of massive young stellar objects (MYSOs). The spatial compactness and the narrow linewidth (owing to the required high degree of velocity coherence) make maser emission an ideal tool for testing ordered velocity fields from the inner protostellar cocoon $\left(\lesssim 10^{3} \mathrm{AU}\right)$. At typical distances of a few kpc for MYSOs, mas-resolution (i.e., Very Long Baseline Interferometry, VLBI) observations of the spatial distribution of each masing cloudlets (as small as a few AU) monitored over time allows us to measure the full-space kinematics of the masing gas. In particular, those sites exhibiting multiple molecular masers gain greater interest since different molecular species can trace distinct and complementary physical environments, thus providing us with information useful to distinguish among different dynamical processes such as rotation, expansion, or contraction.

Almost a decade ago, we started a campaign of multi-epoch, VLBI observations of the three most powerful maser transitions, of water $\left(\mathrm{H}_{2} \mathrm{O}\right)$ at $22.2 \mathrm{GHz}$, methanol $\left(\mathrm{CH}_{3} \mathrm{OH}\right)$ at $6.7 \mathrm{GHz}$, and hydroxyl $(\mathrm{OH})$ at $1.665 \mathrm{GHz}$ toward HMSFRs already well studied with interferometers in thermal, continuum and line emission (e.g., Moscadelli et al. 2007,2011; Goddi et al. 2007,2011; Sanna et al. 2010a,b). Our idea was to complement 


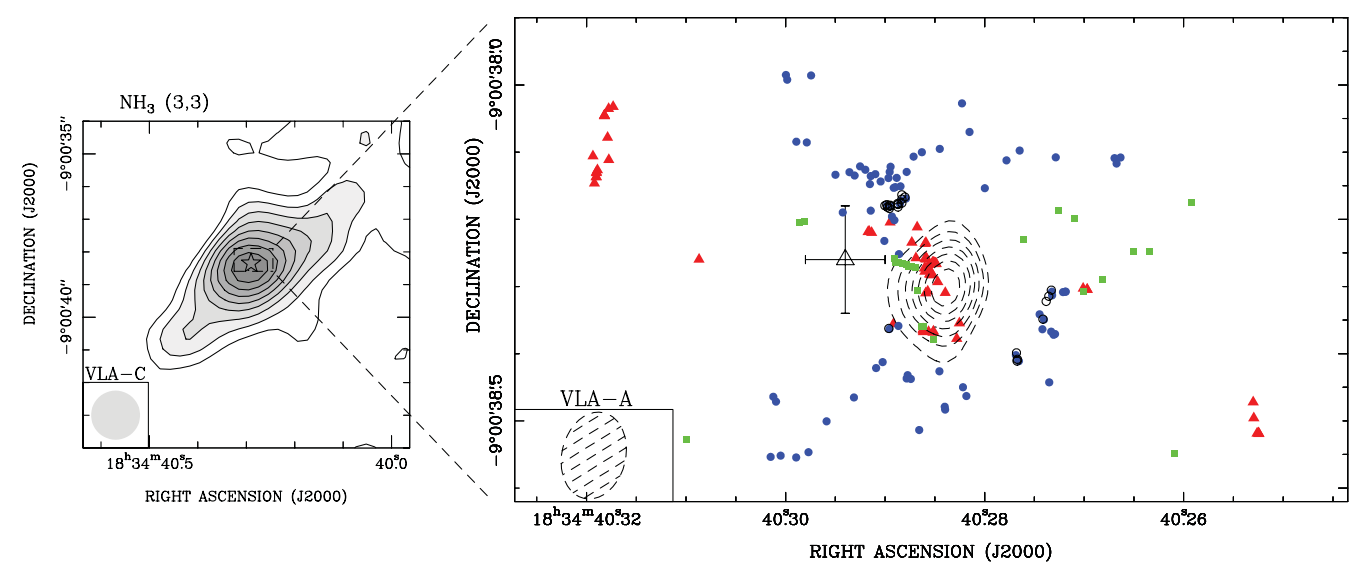

Figure 1. Collection of the subarcsec observations toward G23.01-0.41. Left panel: map of the $\mathrm{NH}_{3}(3,3)$ line from Codella et al. (1997) with contour levels at multiples of $10 \%$ of the peak emission $\left(46 \mathrm{mJy}_{\text {beam }}^{-1}\right)$. The star marks the peak position of the $3 \mathrm{~mm}$ continuum emission as determined by Furuya et al. (2008). Right panel: zoom in on the maser emitting region. Red triangles, blue dots, and green squares represent respectively $\mathrm{H}_{2} \mathrm{O}, \mathrm{CH}_{3} \mathrm{OH}$, and $\mathrm{OH}$ maser positions from our VLBI measurements (Sanna et al. 2010b). The empty circles mark the position of the $12.2 \mathrm{GHz} \mathrm{CH}_{3} \mathrm{OH}$ maser cloudlets (Sanna et al. in prep.). The empty triangle with the error bars indicates the position (and the associated uncertainty) of the $4.8 \mathrm{GHz} \mathrm{H}_{2} \mathrm{CO}$ maser feature derived by Araya et al. (2008). Dashed contours show the $1.3 \mathrm{~cm}$ continuum emission with contour levels at multiples of $10 \%$ of the peak emission $\left(0.72 \mathrm{mJy}^{\mathrm{beam}}{ }^{-1}\right)$.

the information from thermal tracers of outflows (e.g., $\mathrm{CO}$ and $\mathrm{SiO}$ ) and hot molecular cores (HMCs) e.g., dust continuum, $\mathrm{NH}_{3}$, and $\mathrm{CH}_{3} \mathrm{CN}$ ), at typical scales from about $0.1 \mathrm{pc}$ to several $1000 \mathrm{AU}$, with that on the inner few $1000 \mathrm{AU}$ from the (proto)star, provided by maser emission and radio continuum from shock-induced ionization (i.e., thermal jets/winds) or photoionization by the central MYSO (i.e., young H II regions).

\section{An overview of G23.01-0.41}

G23.01-0.41 is an active site of massive star formation $\left(\mathrm{L}_{\mathrm{bol}} \sim 10^{5} \mathrm{~L}_{\odot}\right)$ with a copious amount of maser emission from different molecular species (see Tab. 1). This star-forming region has an accurate distance measurement of $4.6 \pm 0.4 \mathrm{kpc}$, determined through the trigonometric parallax of the associated $12 \mathrm{GHz}$ methanol masers (Brunthaler et al. 2009; see also the chapter by Reid et al.), and belongs to a larger star-forming complex possibly triggered by the close supernova remnant W41 (Leahy \& Tian 2008). On a pc scale, the $4.5 \mu \mathrm{m}$ excess of the Spitzer IRAC GLIMPSE observations (Benjamin et al. 2003; see also Cyganowski et al. 2009, their Figure 1) show a shocked molecular clump powered by a massive, ${ }^{12} \mathrm{CO}$ bipolar outflow detected with the IRAM interferometer by Furuya et al. (2008). Because of the small width-to-length ratio of both the blueshifted and redshifted outflow lobes (to the NE and SW, respectively), such a geometry indicates that the inclination angle of the outflow axis with respect to the plane of the sky must be rather small (particularly evident in our new SMA observations; Sanna et al. in prep.). Following the statistical analysis by Cyganowski et al. (these proceedings), the association of G23.01-0.41 with such an extended green object (EGO) suggests an enhanced outflow activity at an early stage of massive star formation, and thus (presumably) an actively accreting MYSO. At ten-times smaller scale, $\mathrm{CH}_{3} \mathrm{CN}$ and $\mathrm{NH}_{3}$ thermal emissions reveal an $\mathrm{HMC}$ of $70 \mathrm{M}_{\odot}$ with a flattened structure and a velocity gradient $\left(\sim 1 \mathrm{~km} \mathrm{~s}{ }^{-1}\right.$ over 0.1 pc) along its major axis (Furuya et al. 2008; Codella et al. 1997). Since the HMC is 
Table 1. Overall maser detections/upper limits toward G23.01-0.41

\begin{tabular}{cccccc}
\hline Molecule & $\begin{array}{c}\nu \\
(\mathbf{G H z})\end{array}$ & Telescope & $\begin{array}{c}\mathbf{F}_{\text {peak }} \\
(\mathrm{Jy})\end{array}$ & $\begin{array}{c}\mathbf{V}_{\text {LSR }} \\
\left(\mathrm{km} \mathrm{s}^{-1}\right)\end{array}$ & Ref. $^{{ }^{1}}$ \\
\hline $\mathrm{H}_{2} \mathrm{O}$ & 22.2 & VLBA & 320 & 72.6 & 1 \\
\hline $\mathrm{OH}$ & 1.612 & Nançay Radio tel. & 0.2 & 78 & 2 \\
& 1.665 & VLBA & 2.6 & 67.9 & 1 \\
& 1.667 & Nançay Radio tel. & 9 & 75 & 2 \\
\hline $\mathrm{CH}_{3} \mathrm{OH}$ & 6.7 & EVN & 440 & 74.7 & 1 \\
& 12.2 & VLBA & 20 & 75.0 & 3 \\
& 19.9 & Tidbinbilla $70-\mathrm{m}$ & $<0.26$ & & 4 \\
& 23.1 & Parkes 64-m & $<0.9$ & & 6 \\
& 37.7 & Mopra 22-m & $<0.9$ & & 6 \\
& 38.3 & Mopra 22-m & $<0.9$ & & 7 \\
& 38.5 & Mopra 22-m & $<0.9$ & 77.1 & 8 \\
& 44.1 & Parkes 64-m & 40 & & 9 \\
& 85.5 & SEST & $<3.8$ & 77.4 & 10 \\
& 95.2 & Mopra 22-m & 3 & 75.9 & 10 \\
\hline & 107.0 & SEST & 5.2 & & 12 \\
\hline $\mathrm{H}_{2} \mathrm{CO}$ & 108.8 & Mopra 22-m & $<5.1$ & & \\
& 156.6 & SEST & $<2$ & 73.6 & \\
\hline
\end{tabular}

Notes:

$\mathrm{F}_{\text {peak }}$ and $\mathrm{V}_{\mathrm{LSR}}$ are the peak flux density and its Local Standard of Rest velocity for each maser transition, respectively.

1 Ref.: (1) Sanna et al. (2010b); (2) Szymczak \& Gérard (2004); (3) Brunthaler et al. (2009); (4) Ellingsen et al. (2004); (5) Cragg et al. (2004); (6) Ellingsen et al. (2011); (7) Slysh et al. (1994); (8) Cragg et al. (2001); (9) Val'tts et al. (2000); (10) Caswell et al. (2000); (11) Val'tts et al. (1999); (12) Araya et al. (2008).

close to the center of the bipolar outflow and is elongated perpendicular to the outflow axis, it has been interpreted as a candidate rotating toroid, i.e. a massive, non-equilibrium structure accreting onto the embedded (proto)star(s) over a timescale shorter than its rotation period.

All the strongest maser species known to date are observed to cluster at the center of the HMC together with faint continuum emission at centimeter wavelengths (Sanna et al. 2010b; Brunthaler et al. 2009; Araya et al. 2008). The spectral index of the radio continuum is consistent with shock excited emission from a thermal jet, whereas its spatial morphology at the longest VLA baselines clearly draws a bright knot along the NE-SW direction of the molecular outflow (Sanna et al. 2010b). Between 2005 and 2007, we conducted phase-referencing, VLBA and EVN observations of the $\mathrm{H}_{2} \mathrm{O}, \mathrm{CH}_{3} \mathrm{OH}$ (both at several epochs), and of the $\mathrm{OH}$ masers (at a single epoch) in the K, C, and L bands, respectively (see Tab. 1). Recently, we have also complemented this data set with archival VLBA observations in the $\mathrm{U}$ band, in order to study the details of the methanol maser kinematics at $12 \mathrm{GHz}$ with respect to the $6.7 \mathrm{GHz}$ transition (Sanna et al. in prep.). These observations reveal that all the maser emissions in the region participate in an overall expansion from a common center, which possibly denotes the position of the YSO (Sanna et al. 2010b). Furthermore, these maser species sample different positions in the gas around G23.01-0.41 and their radial and transverse velocities pinpoint 3 distinct kinematical structures (see Fig. 1). The $\mathrm{H}_{2} \mathrm{O}$ masers trace both: I) shocked gas along the route of the same bipolar jet exciting the radio continuum and II) a wide-angle, faster wind projected close to the putative position of the YSO (where $\mathrm{H}_{2} \mathrm{O}$ masers are also flaring; see Figure 5b of Sanna et al. 2010b). Similarly, the $\mathrm{OH}$ masers projected close to the fast-moving water masers are strongly blueshifted with respect to the systemic velocity of the region (Figure 3c of Sanna et al. 2010b). We have hypothesized that: I) this emission may be tracing an expanding layer of gas at a larger distance from the YSO 
than the $\mathrm{H}_{2} \mathrm{O}$ gas and II) the same wide-angle wind responsible for the fast water maser shocks could power the expansion of the $\mathrm{OH}$ gas. Finally, the spatial distribution and velocity field of the $\mathrm{CH}_{3} \mathrm{OH}$ cloudlets sketch out a funnel-like structure that probably results from a combination of both rotation and expansion inside the $\mathrm{NH}_{3}$ toroid (Figure 6 of Sanna et al. 2010b).

\section{Details on the Maser Emission}

Water masers. It is interesting to note that, the momentum rate of the water maser jet can be directly compared with that of the large-scale ${ }^{12} \mathrm{CO}$ outflow. This calculation shows consistent values of a few $0.1 \mathrm{M}_{\odot} \mathrm{yr}^{-1} \mathrm{~km} \mathrm{~s}^{-1}$ and has two implications: I) it brings further support to the hypothesis that water masers more detached from the radio continuum trace the primary wind (i.e., the jet) driving the large-scale molecular outflow; II) provided that molecular outflows are momentum driven and their momentum rate is correlated with the mass and luminosity of the YSO (e.g., Figure 4 of Beuther et al. 2002), the high value measured above implies indeed a ZAMS star of early B/late O spectral type. Furthermore, the detection of a wide-angle wind from water masers close to the MYSO can explain the observation of a width-to-length ratio for the molecular outflow of $\sim 0.4-0.3$, much higher than in a pure jet-driven outflow.

Methanol masers. Focusing on the $6.7 \mathrm{GHz}$ maser transition, by observing 3 different EVN epochs spanning 2 years, we have measured for the first time the internal proper motions of individual, methanol maser cloudlets with relative uncertainties less than 30\% (together with the source G16.59-0.05 from Sanna et al. 2010a). The line-of-sight velocity distribution is clearly bipolar, showing two distinct groups of methanol masers, one moving away from the observer (southern redshifted cluster) and one toward the observer (northern blueshifted cluster). By studying the distribution of the 3-dimensional methanol maser velocities with respect to the jet direction on the sky and its inclination with respect to the l.o.s., we have proposed that the methanol gas undergoes rotation about the jet axis and is simultaneously dragged into the outflow motion along this axis (Figure 8 of Sanna et al. 2010b). If we assume centrifugal equilibrium, the rotational component inferred from the velocity field of the methanol gas implies again a central, massive ZAMS star with an early B/late O spectral type. A further, detailed comparison of the spatial distribution and kinematics of the strong maser emission at $12 \mathrm{GHz}$ and $6.7 \mathrm{GHz}$ suggests that both transitions are excited from the same methanol gas (Sanna et al. in prep.). According to Cragg et al. (2005), this evidence constrains the gas temperature and density to values of $\mathrm{T}_{\mathrm{k}}=30-50 \mathrm{~K}$ and $\mathrm{n}_{\mathrm{H}_{2}}=10^{6}-10^{7} \mathrm{~cm}^{-3}$, well in agreement with the physical parameters of the ammonia core. This analysis strongly supports the hypothesis that the Class II $\mathrm{CH}_{3} \mathrm{OH}$ masers may emerge from the inner part of the massive toroid traced on a larger scale with the $\mathrm{CH}_{3} \mathrm{CN}$ and $\mathrm{NH}_{3}$ molecules. It is also worth noting that G23.01-0.41 is one of the rare cases where $\mathrm{H}_{2} \mathrm{CO}$ maser emission at $4.8 \mathrm{GHz}$ was recently found by Araya et al. (2008). This emission arising from a region consistent both in position and l.o.s. velocity with the blueshifted methanol masers may, when refined models for $\mathrm{H}_{2} \mathrm{CO}$ pumping mechanism become available (e.g., Araya et al. 2007), help to further constrain the physical conditions in the region.

Evolutionary sequence in G23.01-0.41. Finally, we want to use a recent evolutionary sequence proposed to explain the appearance and relative lifetimes of different maser species in HMSFRs (cf. Figure 6 of Breen et al. 2010; see also Breen et al., these proceedings) to speculate on the relative phase of evolution for G23.01-0.41. First of all, the star-forming region is associated with strong $4.5 \mu \mathrm{m}$ emission (i.e., an EGO) and shows also bright $44 \mathrm{GHz}$ methanol masers along the direction of the outflow (e.g., Cyganowski 
et al. 2009). The presence of Class I methanol masers and strong outflow activity, together with our findings of faint radio continuum emission mostly due to shock-induced ionization, rule out the far side of the Breen's scale, where photoionization from the central MYSO should produce a bright UCH II region. On the other hand, very bright 6.7 GHz and $12 \mathrm{GHz}$ methanol masers (as found in G23.01-0.41) seem to be associated with a somewhat late stage of evolution for MYSO (Breen et al. 2011), that rules out the first half of the Breen's scale as well. The evidence for an intermediate evolutionary phase of G23.01-0.41 is further supported by the simultaneous presence of bright, mainline, $\mathrm{OH}$ masers, that are usually found in the vicinity of a detectable UCH II region. Following this evolutionary sequence, we can speculate that: I) G23.01-0.41 should develop in the near future a visible UCH II region; II) since high accretion rates are the main mechanism proposed for quenching of UCH II regions, G23.01-0.41 should be still actively accreting material from its protostellar envelope, that would be detectable with ALMA observations of thermal, dense gas tracers.

\section{Acknowledgements}

This work has been supported by the ERC Advanced Grant GLOSTAR under grant agreement no. 247078.

\section{References}

Araya, E., Hofner, P., \& Goss, W. M. 2007, IAU Symposium, 242, 110

Araya, E. D., Hofner, P., Goss, W. M., et al. 2008, ApJS, 178, 330

Benjamin, R. A., Churchwell, E., Babler, B. L., et al. 2003, PASP, 115, 953

Beuther, H., Schilke, P., Sridharan, T. K., et al. 2002, A\&A, 383, 892

Breen, S. L., Ellingsen, S. P., Caswell, J. L., \& Lewis, B. E. 2010, MNRAS, 401, 2219

Breen, S. L., Ellingsen, S. P., Caswell, J. L., et al. 2011, ApJ, 733, 80

Brunthaler, A., Reid, M. J., Menten, K. M., et al. 2009, ApJ, 693, 424

Caswell, J. L., Yi, J., Booth, R. S., \& Cragg, D. M. 2000, MNRAS, 313, 599

Codella, C., Testi, L., \& Cesaroni, R. 1997, A\&A, 325, 282

Cragg, D. M., Sobolev, A. M., Ellingsen, S. P., et al. 2001, MNRAS, 323, 939

Cragg, D. M., Sobolev, A. M., Caswell, J. L., Ellingsen, S. P., \& Godfrey, P. D. 2004, MNRAS, 351,1327

Cragg, D. M., Sobolev, A. M., \& Godfrey, P. D. 2005, MNRAS, 360, 533

Cyganowski, C. J., Brogan, C. L., Hunter, T. R., \& Churchwell, E. 2009, ApJ, 702, 1615

Ellingsen, S. P., Cragg, D. M., Lovell, J. E. J., et al. 2004, MNRAS, 354, 401

Ellingsen, S. P., Breen, S. L., Sobolev, A. M., et al. 2011, ApJ, 742, 109

Furuya, R. S., Cesaroni, R., Takahashi, S., et al. 2008, ApJ, 673, 363

Goddi, C., Moscadelli, L., Sanna, A., Cesaroni, R., \& Minier, V. 2007, A\&\&A, 461, 1027

Goddi, C., Moscadelli, L., \& Sanna, A. 2011, A\& $A$, 535, L8

Leahy, D. A. \& Tian, W. W. 2008, AJ, 135, 167

Moscadelli, L., Goddi, C., Cesaroni, R., Beltrán, M. T., \& Furuya, R. S. 2007, A\& A, 472, 867

Moscadelli, L., Cesaroni, R., Rioja, M. J., Dodson, R., \& Reid, M. J. 2011, A\&3A, 526, A66

Sanna, A., Moscadelli, L., Cesaroni, R., et al. 2010a, A\&A, 517, A71

Sanna, A., Moscadelli, L., Cesaroni, R., et al. 2010b, A\& A, 517, A78

Slysh, V. I., Kalenskii, S. V., Valtts, I. E., \& Otrupcek, R. 1994, MNRAS, 268, 464

Szymczak, M. \& Gérard, E. 2004, A\&A, 414, 235

Val'tts, I. E., Ellingsen, S. P., Slysh, V. I., et al. 1999, MNRAS, 310, 1077

Val'tts, I. E., Ellingsen, S. P., Slysh, V. I., et al. 2000, MNRAS, 317, 315 